\title{
-NOTES-
}

\section{NON-SEPARABLE SOLUTIONS OF THE HELMHOLTZ WAVE EQUATION*}

\author{
DONALD S. MOSELEY (Vitro Laboratories, Silver Spring, Maryland)
}

\begin{abstract}
A set of solutions not obtainable by the method of separation of variables is presented for the vector Helmholtz wave equation in circular cylindrical coordinates limited to non-angular dependence. These are constructed of Bessel and trigonometric functions. For example, if $\mathbf{A}$ is the vector, the $r$-component of the simplest member of the set is

$$
A_{r}=C_{1}\left[m r J_{0}(p r) \cos (m z)+p z J_{1}(p r) \sin (m z)\right] e^{-i \omega t},
$$

where $C_{1}$ is an arbitrary constant, $m$ and $p$ are propagation constants, and $\omega$ is angular frequency. Brief reference is made to three-dimensional solutions in rectangular coordinates.

Introduction. Vector and scalar wave equations appear in electrodynamics, in elastodynamics, and in many other problems involving radiation of energy. During an attempt to formulate, in closed form, the natural modes of elastic vibration of an isotropic solid cylinder of traction-free surfaces and finite dimensions, the writer uncovered some non-separable, simple harmonic solutions that are not described in the standard works [1-8] on partial and ordinary differential equations. This brief paper is a presentation of these solutions in circular cylindrical coordinates, as befit the geometry of the elastic problem, and the paper concludes with some remarks about non-separable solutions in rectangular coordinates.
\end{abstract}

In vector form the Helmholtz wave equation is

$$
\left[\nLeftarrow+k^{2}\right] \mathbf{A}=0, \quad k^{2}=(\omega / c)^{2}
$$

where is the vector Laplacian operator, ${ }^{1} \omega$ is $2 \pi$ times the frequency, $c$ is the velocity with which a constant phase is propagated, and A is a field vector. Equation (1) represents three scalar partial differential equations customarily solved by the method of separating variables.

In his elastic problem the writer assumed axial symmetry to avoid dependence upon the angular coordinate. With this simplification the separable-type solutions of Eq. (1) are

$$
\begin{aligned}
& A_{r}=\left\{\begin{array}{c}
J_{1}(p r) \\
N_{1}(p r)
\end{array}\right\}\left\{\begin{array}{c}
\cos m z \\
\sin m z
\end{array}\right\} e^{-i \omega t}, \\
& A_{z}=\left\{\begin{array}{c}
J_{0}(p r) \\
N_{0}(p r)
\end{array}\right\}\left\{\begin{array}{c}
\sin m z \\
\cos m z
\end{array}\right\} e^{-i \omega t},
\end{aligned}
$$

*Received December 12, 1963. This work was supported by the Air Force Office of Scientific Research under Contract AF 49(638)-1148.

'This notation has been introduced in [9]. 
where $A_{r}$ and $A_{z}$ are radial and axial components of particle displacement, $p$ and $m$ are propagation constants related to $\omega$ by the relation

$$
k^{2}=p^{2}+m^{2},
$$

and Neumann-type factors are not admitted because the origin must not be the site of a pole.

Arbitrarily, the writer chose to restrict attention to motions that are symmetric rather than antisymmetric about the midplane of the cylinder. This choice drops $\sin m z$ and $\cos m z$ from Eqs. (2) and (3), respectively, leaving

$$
\begin{aligned}
& A_{r}=C_{0} J_{1}(p r) \cos (m z) e^{-i \omega t}, \\
& A_{z}=D_{0} J_{0}(p r) \sin (m z) e^{-i \omega t} .
\end{aligned}
$$

The foregoing are standard solutions and well known.

Non-Separable Solutions. Preserving all preceding restrictions, namely, the exclusion of Neumann functions and the symmetries about and along the axis, one can demonstrate that the following pair are also solutions of Eq. (1) when Eq. (4) is true:

$$
\begin{aligned}
& A_{r}=C_{1}\left[m r J_{0}(p r) \cos (m z)+p z J_{1}(p r) \sin (m z)\right] e^{-i \omega t}, \\
& A_{z}=D_{1}\left[m r J_{1}(p r) \sin (m z)+p z J_{0}(p r) \cos (m z)\right] e^{-i \omega t} .
\end{aligned}
$$

One can likewise show that the following pair involving $z^{2}$ and $r^{2}$ satisfies the wave equations:

$$
\begin{aligned}
A_{r}=C_{2}\left\{\left[(p z)^{2}+(m r)^{2}\right]\right. & \left.J_{1}(p r) \cos (m z)+p r J_{0}(p r)[\cos (m z)-2 m z \sin (m z)]\right\} e^{-i \omega t}, \\
A_{z}=D_{2}\left\{p\left[(p z)^{2}+(m r)^{2}\right]\right. & J_{0}(p r) \sin (m z) \\
& \left.\left.-r J_{1}(p r)\left[p^{2}+2 m^{2}\right) \sin (m z)+2 p^{2} m z \cos (m z)\right]\right\} e^{-i \omega t} .
\end{aligned}
$$

A generalization of the foregoing solutions of the wave equation is given by

$$
\begin{gathered}
A_{r}=\sum_{n=0}^{\infty} \sum_{q=0}^{\infty} r^{2 n} z^{2 q}\left\{\left[C_{2 n, 2 q} J_{1}(p r)+C_{2 n+1,2 q} r J_{0}(p r)\right] \cos (m z)\right. \\
\left.+\left[C_{2 n, 2 q+1} J_{1}(p r)+C_{2 n+1,2 q+1} r J_{0}(p r)\right] z \sin (m z)\right\} e^{-i \omega t}, \\
A_{z}=\sum_{n=0}^{\infty} \sum_{a=0}^{\infty} r^{2 n} z^{2 q}\left\{\left[D_{2 n, 2 q} J_{0}(p r)+D_{2 n+1,2 q} r J_{1}(p r)\right] \sin (m z)\right. \\
\left.+\left[D_{2 n, 2 q+1} J_{0}(p r)+D_{2 n+1,2 q+1} r J_{1}(p r)\right] z \cos (m z)\right\} e^{-i \omega t},
\end{gathered}
$$

where, for $k^{2}=p^{2}+m^{2}$, the arbitrary constants $C_{i i} \cdots D_{i i}$ obey $8(n+1)(q+1)$ equations, which are indicated by

$$
\begin{gathered}
(2 n+2)(2 n) C_{2 n+2,2 q}+(2 q+2)(2 q+1) C_{2 n, 2 q+2} \\
-2(2 n+1) p C_{2 n+1,2 q}+2(2 q+1) m C_{2 n, 2 q+1}=0 \\
2(2 n+2) p C_{2 n+2,2 q}+2(2 q+1) m C_{2 n+1,2 q+1} \\
+(2 n+4)(2 n+2) C_{2 n+3,2 q}+(2 q+2)(2 q+1) C_{2 n+1,2 q+2}=0 \\
-2(2 q+2) m C_{2 n, 2 q+2}-2(2 n+1) p C_{2 n+1,2 q+1} \\
+(2 n+2)(2 n) C_{2 n+2,2 q+1}+(2 q+3)(2 q+2) C_{2 n, 2 q+3}=0
\end{gathered}
$$




$$
\begin{gathered}
-2(2 q+2) m C_{2 n+1,2 q+2}+2(2 n+2) p C_{2 n+2,2 q+1} \\
+(2 n+4)(2 n+2) C_{2 n+3,2 q+1}+(2 q+3)(2 q+2) C_{2 n+1,2 q+3}=0 \\
(2 n+2)^{2} D_{2 n+2,2 q}+(2 q+2)(2 q+1) D_{2 n, 2 q+2} \\
+2(2 n+1) p D_{2 n+1,2 q}-2(2 q+1) m D_{2 n, 2 q+1}=0 \\
-2(2 n+2) p D_{2 n+2,2 q}-2(2 q+1) m D_{2 n+1,2 q+1} \\
+(2 n+2)^{2} D_{2 n+3,2 q}+(2 q+2)(2 q+1) D_{2 n+1,2 q+2}=0 \\
2(2 q+2) m D_{2 n, 2 q+2}+2(2 n+1) p D_{2 n+1,2 q+1} \\
+(2 n+2)^{2} D_{2 n+2,2 q+1}+(2 q+3)(2 q+2) D_{2 n, 2 q+3}=0 \\
2(2 q+2) m D_{2 n+1,2 q+2}-2(2 n+2) p D_{2 n+2,2 q+1} \\
+(2 n+2)^{2} D_{2 n+3,2 q+1}+(2 q+3)(2 q+2) D_{2 n+1,2 q+3}=0
\end{gathered}
$$

Note as an example that Eq. (9) is a special case of Eq. (11), which results from $n=q=0$ and $C_{01}=C_{12}=C_{21}=C_{30}=C_{03}=C_{31}=C_{13}=0$.

Note also that, from a mathematical point of view, Eqs. (11-20) are less than general, being restricted by conditions of symmetry and of finiteness of function at the origin.

In rectangular coordinates the writer has made an extension to three dimensions for functions typified by Eqs. (7) and (8). In these coordinates we have

$$
\nLeftarrow \mathbf{A}=\mathbf{i} \nabla^{2} A_{x}+\mathbf{j} \nabla^{2} A_{y}+\mathbf{k} \nabla^{2} A_{z},
$$

and the wave equation becomes

$$
\left(\nLeftarrow+k^{2}\right) \mathbf{A}=\left(\nabla^{2}+k^{2}\right)\left(\mathbf{i} A_{x}+\mathbf{j} A_{y}+\mathbf{k} A_{z}\right)=0 .
$$

Set

$$
A_{x}=\left(c_{1} x X_{1} Y_{1} Z_{1}+c_{2} y X_{2} Y_{2} Z_{2}+c_{3} z X_{3} Y_{3} Z_{3}\right) e^{-i \omega t},
$$

where $X_{i}, Y_{i}$, and $Z_{i}$ are functions only of $x, y$, and $z$, respectively. The $x$-component of the wave equation is thus

$$
\begin{aligned}
& \left(\nabla^{2}+k^{2}\right) A_{x}=c_{1} x\left(\frac{X_{1}^{\prime \prime}}{X_{1}}+\frac{Y_{1}^{\prime \prime}}{Y_{1}}+\frac{Z_{1}^{\prime \prime}}{Z_{1}}+k^{2}\right) X_{1} Y_{1} Z_{1} e^{-i \omega t} \\
& +c_{2} y\left(\frac{X_{2}^{\prime \prime}}{X_{2}}+\frac{Y_{2}^{\prime \prime}}{Y_{2}}+\frac{Z_{2}^{\prime \prime}}{Z_{2}}+k^{2}\right) X_{2} Y_{2} Z_{2} e^{-i \omega t}+c_{3} z\left(\frac{X_{3}^{\prime \prime}}{X_{3}}+\frac{Y_{3}^{\prime \prime}}{Y_{3}}+\frac{Z_{3}^{\prime \prime}}{Z_{3}}+k^{2}\right) X_{3} Y_{3} Z_{3} e^{-i \omega t} \\
& +2\left(c_{1} X_{1}^{\prime} Y_{1} Z_{1}+c_{2} X_{2} Y_{2}^{\prime} Z_{2}+c_{3} X_{3} Y_{3} Z_{3}^{\prime}\right) e^{-i \omega t}=0
\end{aligned}
$$

and the conditions are that the four parentheses on the right of Eq. (24) be separately zero for all $x, y$, and $z$. The first three are identical in form to the equation encountered in the ordinary development of a solution by the method of separation of variables. The fourth prohibits some solutions allowed by the first three, and, in addition, acts as one equation of constraint upon the arbitrary constants $c_{i}$.

Significance. The potential of these non-separable solutions for solving boundary value problems is as yet unassessed and untested, but should emerge as their properties and their applications are more fully explored. 
Acknowledgements. The author is pleased to acknowledge review of the manuscript of this paper and helpful suggestions thereto by Dr. H. M. Trent of the U. S. Naval Research Laboratory and Dr. D. E. Spencer of the University of Connecticut.

\section{REFERENCES}

1. E. T. Whittaker and G. N. Watson, A Course of Modern Analysis, (MacMillan Co., New York, 1948), American edition

2. H. Bateman, Partial Differential Equations of Mathematical Physics, (Dover Publications, Inc., New York, 1944), first American edition

3. E. Kamke, Differentialgleichungen Lösungsmethoden und Lösungen, (Chelsea Publishing Co., New York, 1942)

4. A. G. Webster, Partial Differential Equations of Mathematical Physics, (Dover Publications, Inc., 1955), reprint of second corrected edition

5. A. Sommerfeld, Partial Differential Equations in Physics, (Academic Press, New York, 1949)

6. M. G. Salvadori and R. J. Schwarz, Differential Equations in Engineering Problems, (Prentice-Hall, Englewood Cliffs, New Jersey, 1954)

7. P. Moon and D. E. Spencer, Foundations of Electrodynamics, (D. Van Nostrand Co., Inc., Princeton, New Jersey, 1960)

8. P. Moon and D. E. Spencer, Field Theory for Engineers, (D. Van Nostrand Co., Inc., Princeton, New Jersey, 1961)

9. P. Moon and D. E. Spencer, J. Franklin Inst., 256 (1953) 551

\section{ON LINEAR EQUATIONS OF ANISOTROPIC ELASTIC PLATES*}

\section{BY YI-YUAN YU (Polytechnic Institute of Brooklyn)}

1. Introduction. In a previous paper [1] a generalized Hamilton principle and the associated variational equation of motion are presented for finite elastic deformations. The principle is a generalization of the ordinary Hamilton principle in which only variations of the displacements are admitted. It is also a generalization of the variational principle in elastostatics due to $\mathrm{Hu}[2]^{* *}$ and Washizu [3], and is similar to the latter in that in both principles variations of the displacements, strains, and stresses are taken independently and simultaneously. Various complete systems of equations of nonlinear elastodynamics have been shown to be obtainable as the Euler equations of the generalized Hamilton principle.

In this note the generalized Hamilton principle and the associated variational equation of motion for linear and anisotropic elastic plates are deduced from their counterpart in general elasticity theory given in the previous paper [1], through expansion of the displacement and strain in infinite power series in the manner of Cauchy and Mindlin $[4,5]$ and by carrying out the integration in the thickness direction of the plate. The Euler equations of the variational principle then yield the complete system of plate equations of all orders as were obtained by Mindlin by a different procedure. Some zeroorder and first-order equations for an isotropic plate have been derived before [1] from the generalized variational equation of motion.

${ }^{*}$ Received February 3, 1964. This research was supported by the Air Force Office of Scientific Research of the Office of Aerospace Research, under Contract No. AF 49(638)-1290.

**The author is indebted to Professor C. S. Hsu for drawing attention to Hu's work. 